\title{
CORRESPONDENCE
}

Use of polymerase chain reaction to detect Toxoplasma

Burg et $\mathrm{al}^{1}$ and Savva and Holliman ${ }^{2}$ recently described the use of the polymerase chain reaction (PCR) to detect Toxoplasma gondii. We have also been using amplification reactions to detect the presence of this parasite in clinical specimens since 1989.

Our method is different from that described by these authors, being based on a different target gene. We use a ribosomal DNA (rDNA) sequence, initially described by Johnson et al in phylogenetic studies. ${ }^{3}$ Indeed, in eucaryotes the rDNA is frequently repeated and its sequence consists of a patchwork of highly conserved and specific segments. The primers were chosen to match the most specific segment. Due to its property of natural amplification (hundreds of repeats of the gene), the rDNA is an interesting target for very sensitive detection of $T$ gondii in biological fluids by the PCR. The natural amplification of ribosomal RNA has recently been used elsewhere to detect and differentiate species of Plasmodium. ${ }^{4}$ Because RNA is very susceptible to enzyme degradation, a PCR based on rDNA is likely to be much more reliable than one based on CDNA or RNA.

Briefly, an 88 base pair segment of $T$ gondii rDNA is amplified with the synthetic oligonucleotide primers $5^{\prime}$-CCTTGGCCGATAGGTCTAGG and 5' -AGGCATTCGGGTTAAAGATT. Thirty five cycles are performed (denaturation step $95^{\circ} \mathrm{C}$ for 10 seconds, annealing step $64^{\circ} \mathrm{C}$ for 30 seconds, synthesis step $70^{\circ} \mathrm{C}$ for 60 seconds) as described. ${ }^{5}$ The product can be visualised directly on a polyacrylamide gel and stained with ethidium bromide.

The identity of the amplified molecules can be proved by restriction analysis or Southern blot analysis. Particularly, the 88 base pair PCR product can be controlled by Nsil restriction, providing 35 and 53 base pair fragments. As for hybridisation using a radiolabelled internal probe, we can detect one copy of the rDNA gene ( 0.01 parasites). The higher sensitivity of this procedure avoids having to use hybridisation to detect the presence of $T$ gondii in clinical samples. In fact, we can detect just a single parasite in a buffer solution, and less than five parasites in a mixture of human cells, and the detection is completed within six hours.

We have used this PCR to examine 80 amniotic fluid samples from pregnant women. The results were positive in 10 cases, showing the superiority of this technique over amniotic fluid cultures (four positive) or fetal blood examination (eight positive). For the remaining two, infection was confirmed after birth. There were no false positive results in this prospective trial. Nor were there any false negative results: the PCR tests for the other 70 samples were negative just like the prenatal diagnostic tests and those at one year follow up.

An additional feature is the ability to combine the sensitivity of rDNA detection with the specificity of $\mathrm{P} 30$ detection. The single copy P30 gene encodes for the major surface antigen of $T$ gondii, a membranous tachyzoite protein that is apparently conserved in most

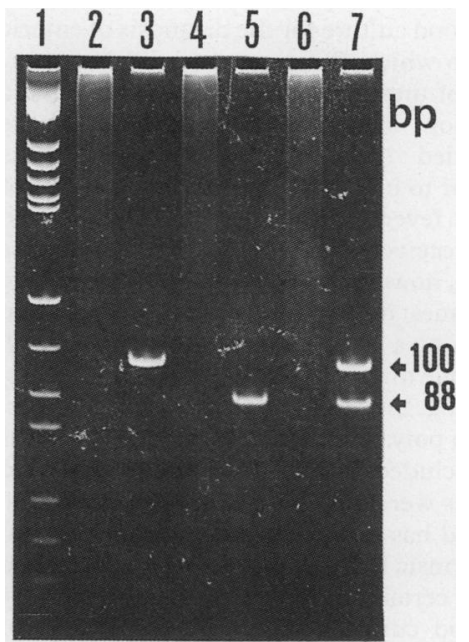

Analysis of amplified products after ethidium bromide staining on $10 \%$ acrylmide gel. Lane 1: PBR 322/HaIII molecular weight marker.

Lanes 2, 4, 6: 200 ng human DNA negative controls with, respectively, $r D N A, P 30$, and rDNA/P30 primers.

Lanes 3, 5, 7:1 pg T gondii DNA diluted in 200 ng human DNA positive reactions, with respectively, $r D N A, P 30$, and $r D N A / P 30$ primers.

strains. Coamplification is achieved in a $100 \mu \mathrm{l}$ reaction buffer $(50 \mathrm{mM} \mathrm{KCl} ; 10 \mathrm{mM}$ TRIS$\mathrm{HCl}$, pH 8.3; $20 \mathrm{mM} \mathrm{MgCl2;} 200 \mu \mathrm{M}$ each dATP, dCTP, dTTP, dGTP) with $0.5 \mu \mathrm{M}$ rDNA specific primers and $0.5 \mu \mathrm{M}$ P30 specific primers: $5^{\prime}$-GCTCCTTGATTCCTGAAGCA 5' -GGGAACTTCTCGATTGGAAC. Two units of Taq Polymerase (Perkin Elmer-Cetus) are added for a 35 cycle reaction. The PCR procedure was as already described, except for the annealing temperature, which was optimised and fixed at $55^{\circ} \mathrm{C}$. The figure shows the PCR products obtained by an rDNA or $\mathrm{P} 30$ reaction and by rDNA/P30 coamplification. No interference is observed in this twin test which remains highly sensitive, and is more specific. The 100 base pair PCR product can be controlled by HinfI restriction analysis (providing 42 and 58 base pair fragments), and by Southern blot analysis.

Studies to determine the efficacy of these tests for antenatal diagnosis and other applications, such as cerebrospinal fluid from patients with AIDS, are in progress.

J CAZENAVE CHEYROU P BLOUIN Medical Laboratory $L A B M$ Department of Molecular Biology, 30 Allées de Tourny 33080 Bordeaux Cedex France AM JOHNSON

Department of Microbiology and Infectious Diseases, Flinders University, South Australia J BEGUERET Department of Genetics, CNRS (UA 542) Bordeaux II University,
France

1 Burg JL, Grover CM, Pouletty P, Boothroyd JC. Direct and sensitive detection of a pathogenic protozoan, Toxoplasma gondii, by
PCR. J Clin Microbiol 1989;27:1787-92.
2 Savva D, Holliman RE. Diagnosis of toxoplasmosis using DNA probes. J Clin Pathol 1990, 43:260-2

3 Johnson AM, Murray PJ, Illana S, Baverstock PJ. Rapid nucleotide sequence analysis of the Jmall subunit ribosomal rDNA of ToxoNA of Toxoplasma gondii: evolutionary implications for the Apicomplexa. Mol Biochem Parasitol 987;25:239-46

4 Waters AP, McCutchan TF. Ribosomal RNA: nature's own polymerase amplified target for diagnosis. Parasitology Today 1990;6:56-9.

5 Cazenave J, Bessieres MH, Sourrue D, Cheyrou A, Begueret J. Detection de toxoplasmes par amplification d'ADN. Revue Française Laboratoires 1990;209:118-26.

\section{Giant cell arteritis}

The paper by Wawryk, Ayberk, Boyd and Rode $^{1}$ provides interesting new data to support the role of adhesion molecules in the development of the inflammatory infiltrate and the granulomatous response in giant cell (temporal) arteritis.

The article began with the premise that the aetiology of giant cell arteritis is unknown and indicates that no antigen (foreign or self) has been defined. These statements do not do justice to what has been written on this subject. Although the authors do allude, in passing, to the studies of Wilkinson and Russell in 1972, they seem to ignore their own observation of a close relation between CAM positive macrophages and the internal elastic lamina in affected vessels (fig 2 of their paper).

We would like to draw attention to the larger body of evidence which supports the view that elastic tissue changes may be the source of the primary antigen in temporal arteritis and that actinic damage may constitute the primary insult responsible for generating or liberating an autoantigen derived from elastic tissue.

The American College of Rheumatology Subcommittee ${ }^{2}$ notes that "a granulomatous inflammatory process is seen that is usually focused along the internal elastic lamina". Identification of the elastic lamina as the probable source of the antigen has been promoted by many observers-for instance, by Healey and Wilske, ${ }^{3}$ Mowat and Hazleman, ${ }^{4}$ and Bengtsson. ${ }^{5}$ The concept that the inflammatory reaction is secondary to primary changes within the lamina is supported by the autoimmune studies of $\mathrm{Cid}$ et al.

That the specific fault in the lamina is actinic elastotic degeneration (solar, basophilic, or "senile" degeneration) was suggested by one of us in 1978, and further evidence has been gathered to support this in later reviews. ${ }^{89}$ The demonstration of actinic degeneration, which occurs classically in the elastic tissue of "photoageing" skin that has been exposed to the sun, is notoriously uncertain in routine histological studies using standard haematoxylin and eosin stains. In sections stained with Harris's haematoxylin under controlled conditions with buffered eosin, however, elastic tissue that has been damaged by radiation stains a distinct blue colour; normal elastic tissue stains a contrasting red colour. ${ }^{89}$ Such changes are more advanced in the superficial aspect of the internal elastic lamina of exposed temporal arteries. ${ }^{89}$ Electron microscopic examination confirms that actinic elastotic degeneration is a prominent feature of affected elastic laminae in temporal arteries exposed to sunshine. 
The temporal arteritis/polymyalgia rheumatica syndrome is a relatively common disorder that is regularly stated to be of unknown aetiology. We respectfully submit that its likely actinic basis is supported by sound observations and deserves wider recognition.

JP O'BRIEN Pathology Laboratory, Wales Medical Centre, Sydney Defartment of Dermatology, Repatriation General Hospital, Sydne EG CLEARY

Department of Pathology, (iniversity of Adelaide, Australia

1 W'awryk SO, Ayberk H, Boyd AW, Rode J Analysis of adhesion molecules in the immunopathogenesis of giant cell arteritis $J$ Clin Fathol 1991;44:497-501.

2 Hiunder GG and Members of the American College of Rheumatology Subcommittee on classification of vasculitis. The American classification of vasculitis. The American College of Rheumatology 1990 criteria for the classification of giant cell

3 Healey LA, Wilske KR. The systemic manifesta tions of temporal arteritis. New York: Grun and Stratton, 1978.

4 Mowat AG, Hazleman BL. Polymyalgia rheu matica-a clinical study with particula reference to arterial disease. J Rheumatol 1974;1:190-202.

5 Bengtsson B-A. Giant cell arteritis. Curren Opinion in Rheumatology 1990;2:60-5.

6 Cid MC, Campo E, Ercilla G, et al. Immunohistochemical analysis of lymphoid and macrophage cell subsets and their immunomacrophage cell subsets and their immunologic activation markers in tempor

7 O'Brien JP. A concept of diffuse actinic arteritis: the role of actinic damage to elastin in 'age change' and arteritis of the temporal artery change' and arteritis of the temporal artery and in polymy

8 O'Brien JP. Destruction of elastic tissue (elas tolysis) as a link between atherosclerosis and the temporal arteritis/polymyalgia rheumatic syndrome: observations on an actinic factor in vascular disease. Pathol Biol 1984;32:123-38.

9 O'Brien JP, Regan W. A study of elastic tissue and actinic radiation in "aging," temporal arteritis, polymyalgia rheumatica, arteritis, polymyalgia rheumatica, and atherosclerosis. the actinic storm in the 24:765-76.

Drs Wawryk et al comment:

O'Brien et al make the interesting suggestion that giant cell arteritis represents an autoimmune response against actinically altered arterial elastic lamina. The authors correctly identify a close relation between macrophages and the internal elastic lamina, as illustrated in our paper. We consistently found that macrophages which express p150/95 and are found in close apposition to the internal elastic lamina strongly expressed ICAM-1 and HLA-DR. A granular pattern of staining for these markers was also seen along the elastic lamina in affected arteries, possibly representing the expression of these molecules on dendritic processes ramifying along the elastic lamina. The functional relation between macrophages showing this phenotype and the elastic lamina, however, and in particular actinically damaged elastic tissue, remains uncertain.

Blood and bone marrow cultures in enteric fever

Dr Farooqui and colleagues present data which support the conclusion that bone marrow culture gives a higher yield than blood culture in patients with enteric fever Although we agree in general with their suggestion that "bone marrow culture could confirm a diagnosis of typhoid fever in patients whose blood cultures are negative," we wish to make some further observations.

There is a considerable body of published work which compares bone marrow culture with blood culture for the diagnosis of enteric fever to which Farooqui et al did not refer. Many of these studies were summarised at a workshop in $1984 .^{2}$ Most workers have concluded that bone marrow culture is superior to blood culture for the diagnosis of enteric fever, particularly in patients who liave received antibiotics. None of these studies, however, used optimal blood culture techniques; most compared a single set, often containing a small volume of blood $(2-3 \mathrm{ml})$, with bone marrow culture. In several studies, including that of Farooqui and colleagues, sodium polyanethol sulphonate (liquoid) was not included in the culture broth, and cultures were only incubated for seven days. Liquoid has been shown to antagonise both the intrinsic bactericidal activity of blood and that of certain antibiotics, ${ }^{3}$ while subculture of blood cultures after the seventh day of incubation may occasionally yield Salmonella typhi. ${ }^{4}$ Farooqui et al mention the possible effect of antibiotics on blood cultures, but they present no data on the previous treatment of their patients.

Our own data, obtained during studies of the antibiotic treatment of typhoid in Kathmandu, Nepal, are shown in the table. On admission to the studies, three blood culture sets $(5 \mathrm{ml}$ blood in $50 \mathrm{ml}$ brain hear infusion broth containing liquoid; Gibco UK) were collected at least 15 minutes apart. Bone marrow $(0.5-1 \mathrm{ml})$ was collected into $20 \mathrm{ml}$ of the same medium. Although the numbers are small, the results show that blood cultures may be positive when bone marrow is negative, and vice versa. Two of the three patients with positive bone marrow and negative blood cultures had received antibiotics (chloramphenicol and cotrimoxazole) within the preceding three days. In two blood culture positive cases at least one blood culture set was negative.

We believe that further studies of the many possible variables are necessary before it is known whether bone marrow culture is superior to blood culture for the diagnosis of enteric fever. At present, we regard the two techniques as complementary. We would therefore disagree with the approach suggested by Farooqui and colleagues-that is, that bone marrow should be cultured in suspected cases of enteric fever only when blood culture is negative after three to four lays of incubation. To optimise the yield of bacteriological investigation, we suggested that, whenever possible, both blood and bone marrow should be cultured when patients with suspected enteric fever are admitted.

Results of paired blood and bone marrow culture from 30 patients with suspected enteric fever

\begin{tabular}{lll}
\hline Blood & Bone marrow & Number \\
\hline Positive & Positive & 12 \\
Positive & Negative & 2 \\
Negative & Positive & 3 \\
Negative & Negative & 13 \\
\hline
\end{tabular}

Of positive cultures, $14 \mathrm{grew} S$ typhi and three grew $S$ paratyphi $A$
The reason most often given for the failure to culture bone marrow is the invasive nature of the procedure. ${ }^{5}$ Bone marrow aspiration with a fine bore needle, however, has been used successfully for the diagnosis of typhoid and is well tolerated. ${ }^{4}$ IE RICHENS Department of Clinical Sciences Lundon School of Hygiene and Tropical Medicine, Keppel Street, condon WCIE $7 H T$

Microbiology and Infectious Diseases, University of Calgary :30 Hospital Drive NW, Calgary, Alberta

Canada T2N 4N

G ACHARYA

B POKHREL

NIR TULADHAR

Tribhudvan University Teaching Hospital, Kathmandu,

1 Farooqui BJ, Khurshid M. Ashfaq MK, Ata Khan $M$. Comparative yield of Salmonella typhi from blood and bone marrow cultures in patients with fever of unknown origin. J Clin Pathol 1991;44:258-9.

2 Edelman R, Levine MM. Summary of an international workshop on typhoid fever. Rev international workshop on

3 Pierce G, Murray PR. Current controversies in the detection of septicemia. Eur J Clin Microbial 1986;5:487-91.

4 West B, Richens JE, Howard PF. Evaluation in Papua New Guinea of a urine coaglutination test and a Widal slide agglutination test for rapid diagnosis of typhoid fever. Trans $R$ Soc Trop Med Hyg 1989;83:715-7.

5 Duthie R, French GL. Comparison of methods for the diagnosis of typhoid fever. J Clin Pathol 1990;43:863-5.

Diagnosis of acute hepatitis $B$ by qualitative assay of specific IgM antibody

Diment ${ }^{\prime}$ disputes my conclusion that qualitative assay of high titre hepatitis B core IgM antibody (anti-HBc IgM) responses has a limited role in the diagnosis of acute hepatitis B surface antigen (HBsAg) positive hepatitis. ${ }^{2} \mathrm{He}$ believes that the disappearance of anti-HBc IgM reactivity in a relatively insensitive assay occurs two months after the onset of acute hepatitis $\mathbf{B}^{1}$ and is therefore manifest as soon as " $\mathrm{e}$ " antigen to " $\mathrm{e}$ " antibody seroconversion takes place. ${ }^{2}$

My observation of anti-HBc IgM persistence beyond " $e$ " antigen " $e$ " antibody seroconversion was considered to reflect use of a particularly sensitive assay. Studies of serial anti-HBc IgM responses in acutely infected patients, however, have not detected loss of antibody until four months, even when the assay used was sufficiently insensitive to give, virtually always, negative results with serum specimens from patients with chronic disease. ${ }^{34}$ Though a relatively insensitive anti-HBc IgM assay may be preferable for diagnosis of acute hepatitis $B$, its use may be confounded by the low titres of antibody occasionally found early in acute hepatitis B. ${ }^{45}$ Thus detection of "e" antigen " $e$ " antibody seroconversion one to two months after onset remains the most certain method of confirming the diagnosis of $\mathrm{HBsAg}$ positive acute hepatitis B.

I agree partly with Dr Howell and colleagues. ${ }^{6} \mathrm{~A}$ presumptive diagnosis of acute hepatitis $B$ can be made by detection of a high titre of $\mathrm{HBsAg}$ in serum by reverse passive haemagglutination (provided false positive 